\title{
1 Diversity reduces extinction risk at global scales
}

3 Authors: Brian C. Weeks ${ }^{1, *}$, Shahid Naeem², Jesse R. Lasky ${ }^{3}$, and Joseph A. Tobias ${ }^{4}$

6 Affiliations:

$7{ }^{1}$ School for Environment and Sustainability, University of Michigan, 440 Church St, Ann Arbor,

8 MI, 48109, USA

$9{ }^{2}$ Ecology, Evolution, and Environmental Biology, Columbia University, 1200 Amsterdam Ave,

10 New York, NY 10027, USA

$11{ }^{3}$ Department of Biology, Pennsylvania State University, University Park, PA, 16802, USA

$12{ }^{4}$ Department of Life Sciences, Imperial College London, Silwood Park, Buckhurst Road, 21

13 Ascot SL5 7PY, UK

14

$15 *$ Correspondence to: bcweeks@umich.edu

16

17 Keywords: biodiversity, bird traits, conservation status, macroecology, extinction risk

19 Statement of authorship: BW conceived the study and performed the analyses; JT and SN

20 helped develop the conceptual framework; JT provided trait data; JR contributed to the analyses;

21 all authors contributed to writing and revising the manuscript. 
23 Data accessibility statement: Trait data used in this study are published openly in the same

24 volume, and the phylogenetic and distributional data are publicly available.

26 Article Type: Letter

27

28 Number of references: 61

29

30 Number of words in abstract: 143

31

32 Number of words in main text: 3,851

33

34 Number of figures: 3

35

36 Number of tables: 0

37

38 Number of text boxes: 0

39

40 OrcID for Brian Weeks: 0000-0003-2967-2970

41 OrcID Shahid Naeem: 0000-0002-6569-2648

42 OrcID for Jesse Lasky: 0000-0001-7688-5296

43 OrcID for Joseph Tobias: 0000-0003-2429-6179 


\section{Abstract}

46 Increases in biodiversity often lead to greater, and less variable, levels of ecosystem

47 functioning. However, whether species are therefore less likely to go extinct in more diverse

48 systems is unclear. We use comprehensive estimates of avian taxonomic, phylogenetic and

49 functional diversity to characterize the global relationship between multiple dimensions of

50 diversity and extinction risk in birds. We find that more diverse assemblages have lower mean

51 IUCN threat status despite being composed of species with attributes that make them more

52 vulnerable to extinction, such as large body size or small range size. Our analyses also reveal that

53 this reduction of current threat status associated with greater diversity far outweighs the

54 increased risk associated with the accumulation of extinction-prone species in more diverse

55 assemblages. These results suggest that species conservation targets can best be achieved by

56 maintaining high levels of overall biodiversity in natural ecosystems. 


\section{Introduction}

Numerous experimental and observational studies have shown that biodiversity is

60 positively associated with an array of ecosystem functions (Cardinale et al. 2002, 2006; Emmett

61 Duffy et al. 2017). Increasingly, research on biodiversity-ecosystem function (BEF)

62 relationships is revealing that diversity-driven increases in function can boost rates at which

63 nutrients, energy and organic matter flow through an ecosystem (Cardinale et al. 2012), as well

64 as increasing its overall multifunctionality (Soliveres et al. 2016), stability (Tilman et al. 2014)

65 and resilience (Oliver et al. 2015). In addition, increased diversity is associated with reduced

66 rates of species invasion (Naeem et al. 2000; Levine et al. 2004; Fargione \& Tilman 2005; Byun

67 et al. 2013) and lower rates of disease transmission (Becker et al. 2014). These benefits are

68 generally conceptualized at the scale of whole ecosystems, yet it is also possible that they

69 influence the fate of individual lineages by reducing extinction risk (Weeks et al. 2016b).

70 However, the relationship between the diversity of an assemblage and the risk of extinction for

71 its constituent lineages is rarely investigated and remains poorly understood.

A key hindrance to progress is that this question is unlikely to be resolved when

73 biodiversity is measured simply in terms of species richness (i.e. taxonomic diversity).

74 Extinction risk may be more closely associated with other aspects of ecosystems, including

75 functional and phylogenetic components of biodiversity (Naeem et al. 2016). For example,

76 functional traits often perform better than species richness in predicting ecosystem function and

77 stability (Tilman et al. 1997; Hooper et al. 2005), suggesting that extinction risk may be more

78 sensitive to variation in functional diversity. Accounting for the multidimensionality of diversity

79 is also important because different facets of biodiversity can have contrasting responses to

80 environmental change (Chapman et al. 2018) and vary in their predicted relationships with 
81 ecosystem function, as well as the mechanisms underpinning those relationships (Flynn et al.

82 2011; Soliveres et al. 2016). As yet, it has not been possible to account for such

83 multidimensionality in studies of extinction risk because the necessary combination of species-

84 level information on geographical distributions, phylogenetic relationships and detailed

85 functional traits have not generally been available at sufficiently large spatial and taxonomic

86 scales (Naeem et al. 2016).

87 Capitalizing on the availability of comprehensive phylogenetic (Jetz et al. 2012) and

88 distributional data for birds (BirdLife International 2015), we develop a multidimensional metric

89 of avian diversity to explore its association with extinction risk at a global scale. Birds offer an

90 ideal system for this approach because they are distributed worldwide with high quality species-

91 level information on co-occurrence, threat status and-increasingly_-functional traits (Tobias et

92 al. 2020). Using a newly compiled data set of morphological trait measurements from $>40,000$

93 individual birds of $>10,000$ species, representing $>99 \%$ of bird species diversity (Tobias et al.,

94 this issue), we calculate functional richness (Villéger et al. 2008) for avian assemblages based on

95 body mass, beak shape, leg length and tail length. At global scales, these traits provide a

96 powerful index of avian dietary niche and foraging behaviour (Pigot et al. 2020). Our estimation

97 of functional richness therefore focuses on 'effect traits' (i.e. traits that determine the

98 contribution of an individual to ecosystem functioning; Winemiller et al. 2015).

99 Since eco-morphological and life history traits are also linked to conservation status in

100 birds (Tobias \& Pigot 2019), we use them to develop a metric of extinction risk. We assume that

101 increases in body mass and ecological specialization, as well as decreases in geographical range

102 size and dispersal ability, are associated with the increased likelihood that a lineage will go

103 extinct per unit time, as reported in numerous studies (e.g. Bennett \& Owens 1997; Şekercioğlu 
104 et al. 2004; Reinhardt et al. 2005; Lee \& Jetz 2011; Jetz \& Freckleton 2015). We quantify

105 dispersal ability using wing morphology (Claramunt et al. 2012; Sheard et al. 2020), and we

106 estimate specialization based on the trophic diversity of each species' diet (Wilman et al. 2014;

107 Pigot et al. 2020). Because these attributes predict the probability that a species will go extinct,

108 we use our trait-based metric of extinction risk to calculate the collective vulnerability of species

109 in assemblages, or 'assemblage vulnerability' (Weeks et al. 2016b). In other words, assemblages

110 composed of species with small range sizes, low dispersal abilities, large body sizes and high

111 levels of ecological specialization have greater overall vulnerability. Since our calculation of

112 assemblage vulnerability is partly based on the presence of species not currently considered

113 threatened with extinction, but likely to become threatened in the future, it provides a measure of

114 latent extinction risk (i.e., the difference between a species' contemporary extinction risk, and the

115 expected level of risk, given its biology; Cardillo et al. 2006).

116 Although they can theoretically capture collective or latent extinction risk, trait-based

117 metrics provide a relatively crude estimate of contemporary extinction risk (Tobias \& Pigot

118 2019). Thus, we also characterize the mean threat status of assemblages using IUCN Red List

119 status (BirdLife International 2015), an indicator of current conservation priorities widely used in

120 global-scale analyses (Isaac et al. 2007). Previous studies have shown that IUCN Red List status

121 and trait-based predictors of extinction risk are correlated in birds (Tobias \& Pigot 2019), but it

122 is less clear how they are linked to biodiversity. Although the standard prediction based on BEF

123 literature is that biodiversity enhances ecosystem functioning, thereby reducing extinction risk,

124 other factors may complicate the outcome. In particular, if occurrence within diverse

125 assemblages reduces rates of extinction for individual lineages, this may — paradoxically_

126 increase assemblage vulnerability through the survival and accumulation of extinction-prone 
127 species (Weeks et al. 2016b; Fig. 1). These contrasting possibilities set up a potential trade-off

128 whereby increased diversity may have both positive and negative implications from the

129 perspective of biological conservation.

130 Comparing across taxonomic, phylogenetic and functional diversity metrics, we examine

131 the effects of different components of bird diversity on assemblage vulnerability and IUCN

132 threat status (Fig. 2). In addition, to shed light on underlying processes, we use structural

133 equation modeling to quantify the strength of the relationships between bird diversity,

134 assemblage vulnerability and extinction risk. The findings allow us to disentangle the positive

135 and negative effects of biodiversity on contemporary and latent extinction risk, with implications

136 for the prioritization of conservation interventions.

138 Methods

139 Presence-Absence Matrix

140 We developed a $1^{\circ}$ latitude by $1^{\circ}$ longitude resolution occurrence map for terrestrial bird

141 communities, excluding all non-terrestrial cells (those that were $>50 \%$ ocean or $>70 \%$ inland

142 water), and all cells below $60^{\circ} \mathrm{S}$. We then determined species composition of these cells using

143 species range maps at a $10^{2} \mathrm{~km}$ resolution, obtained from BirdLife International, projected onto a

$1441^{\circ}$ latitude by $1^{\circ}$ longitude grid. Species ranges were trimmed to exclude areas where presence

145 was classified as uncertain or extinct. We also omitted areas where species origin was classified

146 as vagrant or uncertain, and where seasonality was classified as passage (i.e. only occurring on

147 migration) or uncertain. Species were considered to be present in a grid cell assemblage if their

148 range covered at least $10 \%$ of a cell (unless a species range was sufficiently small that it did not

149 cover $10 \%$ of any cell, in which case that species was considered to be present in any cell that 
150 overlapped with their range). Any cells with fewer than 7 species were removed, so that each cell

151 had enough taxa to calculate functional richness using 6 traits (Villéger et al. 2008). While

152 species occurring in the same $1^{\circ}$ grid cell do not necessarily interact as a community, the total

153 avian assemblage of each cell serves as an estimate of the complete range of traits and trophic

154 interactions that could potentially contribute to ecological functions with relevance to extinction

155 risk. At global scales, quantification and validation of interspecific interactions is not feasible, so

156 co-occurrence within $1^{\circ}$ cells is routinely used as a proxy for coexistence (e.g. Pigot et al. 2016)

157 or to link biodiversity and ecosystem function (e.g. Duchenne et al. 2020).

\section{Community Diversity Metrics}

160 For each assemblage occupying each grid cell, we calculated species richness, two

161 measures of phylogenetic diversity, and one metric of functional diversity (Fig. 2). Functional

162 diversity was characterized using six ecologically-important functional effect traits (total beak

163 length, beak tip to the anterior edge of the nares, beak width, beak depth, tail length, and tarsus

164 length) measured on museum specimens (Pigot et al. 2020). For each assemblage, we used these

165 traits to calculate functional richness - the volume of the convex hull that bounds the functional

166 trait space (Villéger et al. 2008) — using the 'dbfd' function in the FD R package (Laliberté \&

167 Legendre 2010; Laliberté et al. 2015; R Core Team 2018). All traits were standardized to a mean

168 of zero and standard deviation of one prior to analysis. In the 'picante' package in R (Kembel et

169 al. 2010), we used the 'pd' and 'cophynetic' functions, respectively, to calculate the

170 phylogenetic diversity of each assemblage as (1) the sum of the branch lengths connecting all

171 species in the community - i.e. Faith's phylogenetic diversity index (Faith 1992) — and (2) the

172 mean pairwise phylogenetic distance (Webb et al. 2002) between all species in the community. 
173 The phylogenetic relationships among species were estimated using 1,000 phylogenies taken

174 from the posterior distribution of the Jetz et al. (2012) global phylogeny of birds, with the

175 (Hackett et al. 2008) phylogeny used as a backbone, and including species that were placed by

176 Jetz et al. (2012) using taxonomy. From these phylogenies, we calculated a maximum credibility

177 clade tree using DendroPy (Sukumaran \& Holder 2010) as described in Rubolini et al. (2015).

To calculate the accumulation of species with traits and distributions that make them pre-

181 disposed to extinction, we quantified assemblage vulnerability for each assemblage in the world,

182 based on a modification of the approach taken by Weeks et al. (2016b). All variables were

183 standardized to a mean of zero and standard deviation of one prior to calculation of vulnerability

184 for both species and assemblages. For each species in an assemblage, we calculated a species-

185 specific trait-based vulnerability score ( $\mathrm{V}_{\mathrm{t}}$, eqn 1$)$ based on dispersal ability (measured as hand-

186 wing index (Claramunt et al. 2012) with data from (Sheard et al. 2020)), mass from Tobias \&

187 Pigot (2019), and the degree of specialization (based on the trophic diversity of their diets

188 (Wilman et al. 2014; Pigot et al. 2020):

$$
\mathrm{V}_{\mathrm{t}}=(\text { Mass }+ \text { Inverse Hand-wing Index }+ \text { Ecological Specialization }) / 3
$$

191 We then considered the mean of $V_{t}$ and the inverse of range size (which we considered to be the 192 number of cells in which a species was present) to be each species' vulnerability $\left(\mathrm{V}_{\mathrm{s}}\right)$ :

$$
\mathrm{V}_{\mathrm{s}}=\left(\mathrm{V}_{\mathrm{t}}+\text { Inverse Range Size }\right) / 2
$$


We then calculated the assemblage vulnerability for each assemblage, 'i', as the

196 unweighted mean of the vulnerability scores $\left(\mathrm{V}_{\mathrm{s}}\right)$ for all $(\mathrm{n})$ species in an assemblage:

$$
\mathrm{V}_{\mathrm{ai}}=\left(\mathrm{V}_{\mathrm{s} 1}+\mathrm{V}_{\mathrm{s} 2 \ldots .} \mathrm{V}_{\mathrm{sn}}\right) / \mathrm{n}
$$

199 Thus, larger species with low dispersal abilities, greater ecological specialization, and smaller

200 range sizes would have higher species vulnerability $\left(\mathrm{V}_{\mathrm{s}}\right)$ scores, and assemblages that are

201 composed of species that tended to have higher $\mathrm{V}_{\mathrm{s}}$ scores would have higher assemblage

202 vulnerability $\left(\mathrm{V}_{\mathrm{a}}\right)$ scores.

To characterize the contemporary threat status of each assemblage, we calculated the mean IUCN threat status (BirdLife International 2015) of all species in the assemblage. We

207 converted threat status categories to numeric variables, a commonly used approach for

208 characterizing extinction risk (Isaac et al. 2007), with Least Concern $=1$ and Critically

209 Endangered $=5$. For each assemblage, we then calculated the mean IUCN threat status of its

210 constituent species, and standardized the assemblage-level variable to have a mean of 0 and a

211 standard deviation of 1 , to improve model fitting.

We characterized diversity as a latent variable reflected in the observed (i.e. exogenous,

215 as opposed to latent) covariates: species richness, functional richness, phylogenetic diversity, and

216 mean pairwise phylogenetic distance measures of the species in an assemblage (Fig. 3). This

217 approach is based on the conceptual framework of Naeem et al. (2016) in which diversity is 
218 treated as a multidimensional construct, with each exogenous predictor measured as described in

219 the Community Diversity Metrics section, above. The loading of species richness on diversity

220 was set to 1 in order to define the scale of the latent diversity variable (Rosseel 2012). We then

221 modeled assemblage vulnerability as a function of diversity, and contemporary threat status as a

222 function of diversity and assemblage vulnerability (Fig. 3). Each path coefficient linking two

223 variables was considered to be the direct effect of the predictor variable on the response. The

224 indirect effect of diversity on contemporary threat status (via the effect of diversity on

225 assemblage vulnerability) was calculated as the product of the path coefficient linking diversity

226 and assemblage vulnerability and the path coefficient linking assemblage vulnerability and

227 contemporary threat status. All reported coefficients are standardized.

228 To account for the potential impacts of spatial autocorrelation, we fit the structural

229 equation model using a spatially explicit structural equation modeling approach (Rosseel 2012;

230 Lamb et al. 2014). Because the size of the dataset (15,244 assemblages) precluded the

231 calculation of a comprehensive spatial distance matrix, we randomly subsampled 1,000

232 assemblages from the dataset without replacement and fit the model to the subset of the data. To

233 account for spatial autocorrelation, we fit the SEM using 10 lag distances, with an upper limit of

$23450 \%$ of the total distance between subsampled points, using the 'runModels' function from the

235 SESEM package in R (Lamb et al. 2014; R Core Team 2018). This returns 10 fitted SEMs,

236 which we compared using the comparative fit index (CFI). We then extracted the model

237 statistics, parameters, and parameter significance estimates from the SEM based on the lag

238 distance that produced an SEM with the highest CFI value.

239 We repeated this model fitting on random subsets of the data 1,000 times and calculated

240 the mean parameter estimate for each path across all random subsets. We considered the $2.5 \%$ - 
$97.5 \%$ quantiles to be the confidence interval (CI) of each parameter, and considered parameters

242 to be significant if the CI did not overlap with zero. We assessed overall model fit using the

243 comparative fit index (CFI) and root mean square error of approximation (RMSEA).

\section{Results}

We characterized functional, phylogenetic, and taxonomic diversity for 15,224 avian assemblages worldwide (Fig. 2). In our model, our latent diversity variable had a variance of 1, and positive loadings on all exogenous predictors of diversity: species richness $(\beta=0.96)$,

249 functional richness $(\beta=0.69)$, Faith's phylogenetic diversity $(\beta=1.03)$, mean pairwise

250 phylogenetic distance $(\beta=0.32)$, and all relationships between these exogenous variables and 251 diversity were significant (Fig. 3).

We report average model fit statistics from across 1,000 model fits from random subsets

253 of 1,000 assemblages from our dataset. While metrics of the absolute goodness of fit were 254 relatively low $\left(\mathrm{RMSEA}=0.15, P<0.01 ; \chi^{2}=7,524, \mathrm{df}=8, P<0.01\right)$, this is not particularly

255 surprising given the scope of the dataset, the simplicity of the model, and the tendency for 256 ecological data to be noisy. More importantly, the model had a better fit to the data than a null 257 model: $\mathrm{CFI}=0.97, \mathrm{CI}=0.96-0.98$, with anything over $\mathrm{CFI}=0.90 \mathrm{implying}$ a good fit to the 258 data (Kline 2010).

We found that diversity was significantly positively associated with assemblage

260 vulnerability $(\beta=0.72, \mathrm{CI}=0.68-0.76)$, and also explained a substantial fraction of the variance

261 in assemblage vulnerability (assemblage vulnerability $R^{2}=0.52$ ), indicating that more diverse

262 assemblages were on average more vulnerable (i.e. had higher $\mathrm{V}_{\mathrm{s}}$; eqn 2), associated with latent

263 extinction risk. Conversely, diversity was significantly negatively associated with contemporary 
264 extinction risk, indicating more diverse assemblages had lower mean IUCN threat level $(\beta=$ -

$2650.31, \mathrm{CI}=-0.44--0.17)$. Assemblage vulnerability was positively associated with contemporary

266 threat status, but the effect size was small and the relationship was not significant $(\beta=0.03, \mathrm{CI}=$

$267-0.17-0.23)$. As a result, while diversity had a significant negative direct effect on

268 contemporary extinction risk $(\beta=-0.31)$, it had a contrasting positive indirect effect on

269 contemporary extinction risk $(\beta=0.02$; Fig. 3$)$, but this relationship was not significant. Thus,

270 the indirect negative effect of diversity on contemporary threat, driven by the accumulation of

271 more vulnerable species, may have the potential to partly limit the benefit of diversity in

272 reducing contemporary threats, but this contrasting effect is relatively small and non-significant.

273 The model explained roughly $9 \%$ of the variance in contemporary threat status $\left(R^{2}=0.09, \mathrm{CI}=\right.$

$2740.06-0.13)$

275

276 Discussion

By compiling multiple dimensions of diversity data for the global avifauna, we have

278 shown that species occurring in assemblages with higher levels of diversity have reduced

279 contemporary extinction risk. It may seem intuitive that reduced extinction risk has resulted in

280 increased diversity, particularly over deeper timescales at which declining extinction rates

281 towards the equator have allowed species richness to build up in tropical biota, driving latitudinal

282 diversity gradients (Mittelbach et al. 2007). However, our analyses focus on contemporary and

283 latent extinction risk, a temporal scale less relevant to the effect of diversification or glaciation,

284 and more relevant to the near-term trends determining IUCN Red List status and vulnerability.

285 Recent anthropogenic threats have driven relatively few bird lineages to extinction, but have

286 caused a significant proportion of global avian diversity to be classified as threatened (BirdLife 
287 International 2015). At this temporal scale, our results are more likely to be explained by

288 characteristics of diverse ecosystems, with increases across multiple facets of diversity reflecting

289 a higher level and stability of ecosystem functioning. This may take the form of more complete

290 networks of species interactions and associated processes, or the buffering effect of biodiversity

291 against risks such as invasion or disease (Naeem et al. 2000; Levine et al. 2004; Fargione \&

292 Tilman 2005; Byun et al. 2013; Becker et al. 2014).

While the direct reduction in contemporary extinction risk associated with diversity

294 suggests that species in more diverse assemblages are at lower risk of extinction, this relationship

295 is complicated by the dynamic history of community assembly (Weeks et al. 2016a). For

296 example, reduced extinction pressure may result in the long-term survival of species otherwise

297 prone to extinction, which therefore tend to accumulate in diverse assemblages over time (Weeks

298 et al. 2016b). There is some evidence that this occurs in plants: climatic stability is thought to

299 have reduced extinction risk for rare species, allowing them to persist in climatically stable

300 regions, with the result that climate change and anthropogenic drivers of extinction are now

301 disproportionately impacting rare species in more diverse regions (Enquist et al. 2019).

302 In accordance with the idea that diversity can both decrease short-term and increase long-

303 term vulnerability, we find that the reduction in contemporary extinction risk $(\beta=-0.31)$ is

304 coupled with increased latent extinction risk as measured by assemblage vulnerability $(\beta=0.72)$.

305 This suggests that more diverse communities are composed of many species that are not

306 currently threatened but with attributes associated with higher risk of extinction: poor dispersal

307 ability, large body size, greater ecological specialization, and smaller range sizes. One possible

308 interpretation of this pattern is that attributes associated with increased vulnerability may

309 promote diversification (e.g., reduced dispersal ability can lead to increased diversification rates; 
310 Weeks \& Claramunt 2014). However, the association between our indices of vulnerability and

311 diversification at global scales is weak and mixed (Owens et al. 1999; Tobias et al. 2020),

312 suggesting that their role as drivers of diversification is unlikely to explain our results. Overall,

313 we interpret the elevated vulnerability of diverse assemblages as an outcome of lower rates of

314 extinction for extinction-prone species, suggesting that the long-term consequence of lower

315 contemporary extinction risk is an increase in latent extinction risk.

To understand the overall relationship between biodiversity and extinction risk in natural

317 systems, it is therefore important to disentangle the contrasting effects of diversity on the current

318 survival prospects of individual lineages (reduced short-term risk) from the accumulation of

319 species inherently predisposed to extinction in future (increased long-term risk). When we assess

320 the relationship between assemblage vulnerability and contemporary extinction risk, we find a

321 slight positive association. While this result hints at an indirect mechanism by which biodiversity

322 could ultimately be associated with increased contemporary extinction risk, the relationship

323 between assemblage vulnerability and contemporary extinction risk is relatively weak $(\beta=0.03)$

324 and not significant. Moreover, the increase in contemporary extinction risk associated with

325 increased assemblage vulnerability in more diverse assemblages $(\beta=0.02)$ is an order of

326 magnitude weaker than the direct effect of high diversity in reducing contemporary extinction

327 risk $(\beta=-0.31)$. In other words, the effect of diversity in boosting latent extinction risk is

328 negligible in comparison with its effect in reducing contemporary extinction risk.

Although the variables we use to determine assemblage vulnerability and IUCN threat

330 status are widely considered to be indicators of extinction risk, their connection to extinction rate

331 is complicated (Harcourt 2005). For example, some forms of rarity-beyond restricted range size

332 (Rabinowitz 1981) — might influence IUCN threat status designations without necessarily being 
333 related to extinction rates (Harnik et al. 2012). While directly examining the relationship

334 between diversity and extinction rates would be preferable, doing so is not currently feasible

335 given our knowledge of taxa that have gone extinct is highly incomplete, particularly in relation

336 to their ranges, traits and phylogenetic relationships. Our indices of contemporary extinction risk

337 (IUCN threat status) and assemblage vulnerability may be poor predictors of extinction rates

338 across timescales, which may partially explain the weak and non-significant relationship between

339 assemblage vulnerability and contemporary threat status (Fig. 3).

340 The effects of biodiversity on ecosystem function can be complicated by assembly

341 history (Fukami \& Morin 2003) and temporal scale (Reich et al. 2012). For similar reasons,

342 historical biogeography can alter the relationship between biodiversity and vulnerability (Weeks

343 et al. 2016b). Predicting the effects of future biodiversity loss on ecosystem functioning, and

344 thus threat status, may be further complicated by shifts in the species-specific functioning or

345 abundance of surviving taxa (De Laender et al. 2016). Thus, the balance between diversity-

346 driven reductions in contemporary extinction risk and increases in the number of species

347 inherently sensitive to extinction may be altered according to context, with some diverse

348 communities having higher vulnerability than others as a result of the phenotypic, biogeographic

349 and functional attributes of their constituent species. Future studies should therefore analyze the

350 relationship between diversity and extinction risk in different historical contexts and across a

351 range of spatial and temporal scales.

\section{Conclusions}

354 Considering spatial variation in multiple dimensions of diversity at a global scale, we find

355 that higher diversity is associated with reduced contemporary extinction risk and increased 
assemblage vulnerability in birds. We attribute this general pattern to higher levels of ecosystem

357 functioning in more biodiverse assemblages, which can theoretically moderate immediate

358 extinction risks while also inflating the number of extinction-prone species in a community. We

359 also show that the reduction of extinction risk associated with increased diversity is far stronger

360 than the contrasting increase of risk associated with greater assemblage vulnerability in more

361 diverse assemblages. We conclude that the maintenance of biodiverse communities may be a

362 cost-effective approach to preventing extinction, reducing the longer-term need for expensive

363 single-species conservation interventions.

\section{References}

366 Becker, G.C., Rodriguez, D., Felipe Toledo, L., Longo, A.V., Lambertini, C., Correa, D.T., et al.

367 (2014). Partitioning the net effect of host diversity on an emerging amphibian pathogen.

Proc. R. Soc. B Biol. Sci., 281, 20141796.

369 Bennett, P.M. \& Owens, I.P.F. (1997). Variation in extinction risk among birds: chance or

370 evolutionary predisposition? Proc. R. Soc. B Biol. Sci., 264, 401-408.

371 BirdLife International. (2015). IUCN red list for birds. Available at: http://www.birdlife.org.

$372 \quad$ Last accessed 1 January 2015.

373 Byun, C., de Blois, S. \& Brisson, J. (2013). Plant functional group identity and diversity

374 determine biotic resistance to invasion by an exotic grass. J. Ecol., 101, 128-139.

375 Cardillo, M., Mace, G.M., Gittleman, J.L. \& Purvis, A. (2006). Latent extinction risk and the

376 future battlegrounds of mammal conservation. Proc. Natl. Acad. Sci. U. S. A., 103, 4157-

$377 \quad 4161$.

378 Cardinale, B.J., Duffy, J.E., Gonzalez, A., Hooper, D.U., Perrings, C., Venail, P., et al. (2012). 
Biodiversity loss and its impact on humanity. Nature, 486, 59-67.

380 Cardinale, B.J., Palmer, M.A. \& Collins, S.L. (2002). Species diversity enhances ecosystem

381 functioning through interspecific facilitation. Nature, 415, 426-429.

382 Cardinale, B.J., Srivastava, D.S., Duffy, J.E., Wright, J.P., Downing, A.L., Sankaran, M., et al.

383 (2006). Effects of biodiversity on the functioning of trophic groups and ecosystems. Nature, 443, 989-992.

Chapman, P.M., Tobias, J.A., Edwards, D.P. \& Davies, R.G. (2018). Contrasting impacts of land-use change on phylogenetic and functional diversity of tropical forest birds. J. Appl.

Claramunt, S., Derryberry, E.P., Remsen Jr, J. V \& Brumfield, R.T. (2012). High dispersal ability inhibits speciation in a continental radiation of passerine birds. Proc. R. Soc. B Biol.

Duchenne, F., Thébault, E., Michez, D., Elias, M., Drake, M., Persson, M., et al. (2020). Ecol. Evol., 4, 115-121.

394 Emmett Duffy, J., Godwin, C.M. \& Cardinale, B.J. (2017). Biodiversity effects in the wild are common and as strong as key drivers of productivity. Nature, 549, 261-264.

396 Enquist, B.J., Feng, X., Boyle, B., Maitner, B., Newman, E.A., Jørgensen, P.M., et al. (2019). The commonness of rarity: global and future distribution of rarity across land plants. Sci. $A d v ., 5$, eaaz0414.

399 Faith, D.P. (1992). Conservation evaluation and phylogenetic diversity. Biol. Conserv., 61, 1-10.

400 Fargione, J.E. \& Tilman, D. (2005). Diversity decreases invasion via both sampling and $401 \quad$ complementarity effects. Ecol. Lett., 8, 604-611. 
402

403

404

405

406

407

408

409

410

Flynn, D.F.B., Mirotchnick, N., Jain, M., Palmer, M.I. \& Naeem, S. (2011). Functional and phylogenetic diversity as predictors of biodiversity-ecosystem-function relationships. Ecology, 92, 1573-81.

Fukami, T. \& Morin, P.J. (2003). Productivity-biodiversity relationships depend on the history of community assembly. Nature, 424, 423-426.

Hackett, S.J., Kimball, R.T., Reddy, S., Bowi, R.C.K., Braun, E.L., Braun, M.J., et al. (2008). A phylogenomic study of birds reveals their evolutionary history. Science, 320, 1763-1768.

Harcourt, A.H. (2005). Problems of studying extinction risks. Science, 310, 1276.

Harnik, P.G., Simpson, C. \& Payne, J.L. (2012). Long-term differences in extinction risk among the seven forms of rarity. Proc. R. Soc. B Biol. Sci., 279, 4969-4976.

Hooper, D.U., Chapin III, F.S. \& Ewel, J.J. (2005). Effects of biodiversity on ecosystem functioning: a consensus of current knowledge. Ecol. Monogr., 75, 3-35.

Isaac, N.J.B., Turvey, S.T., Collen, B., Waterman, C. \& Baillie, J.E.M. (2007). Mammals on the EDGE: conservation priorities based on threat and phylogeny. PLoS One, 2, e296.

Jetz, W. \& Freckleton, R.P. (2015). Towards a general framework for predicting threat status of data-deficient species from phylogenetic, spatial and environmental information. Philos. Trans. R. Soc. B Biol. Sci., 370, 20140016.

Jetz, W., Thomas, G.H., Hartmann, K. \& Mooers, A.O. (2012). The global diversity of birds in space and time. Nature, 491, 444-448.

Kembel, S.W., Cowan, P.D., Helmus, M.R., Cornwell, W.K., Morlon, H., Ackerly, D.D., et al. (2010). Picante: R tools for integrating phylogenies and ecology. Bioinformatics, 26, 14631464.

Kline, R.B. (2010). Principles and practice of structural equation modeling. 3rd edn. The 


\section{Guilford Press, New York, NY, USA.}

426 De Laender, F., Rohr, J.R., Ashauer, R., Baird, D.J., Berger, U., Eisenhauer, N., et al. (2016).

427 Reintroducing environmental change drivers in biodiversity-ecosystem functioning

$428 \quad$ research. Trends Ecol. Evol., 31, 905-915.

429 Laliberté, E. \& Legendre, P. (2010). A distance-based framework for measuring functional

430 diversity from multiple traits. Ecology, 91, 299-305.

431 Laliberté, E., Legendre, P. \& Shipley, B. (2015). FD: measuring functional diversity from multiple traits, and other tools for functional ecology. R package version 1.0-12. Available

434 Lamb, E.G., Mengersen, K.L., Stewart, K.J., Attanayake, U. \& Siciliano, S.D. (2014). Spatially 435 explicit structural equation modeling. Ecology, 95, 2434-2442.

436 Lee, T.M. \& Jetz, W. (2011). Unravelling the structure of species extinction risk for predictive 437 conservation science. Proc. R. Soc. B Biol. Sci., 278, 1329-1338.

438 Levine, J.M., Adler, P.B. \& Yelenik, S.G. (2004). A meta-analysis of biotic resistance to exotic plant invasions. Ecol. Lett., 7, 975-989.

440 Mittelbach, G.G., Schemske, D.W., Cornell, H. V., Allen, A.P., Brown, J.M., Bush, M.B., et al. 441 (2007). Evolution and the latitudinal diversity gradient: Speciation, extinction and 442 biogeography. Ecol. Lett., 10, 315-331.

443 Naeem, S., Knops, J.M., Tilman, D., Howe, K.M., Kennedy, T. \& Gale, S. (2000). Plant

444 diversity increases resistance to invasion in the absence of covarying extrinsic factors. 445 Oikos, 91, 97-108.

446 Naeem, S., Prager, C., Weeks, B.C., Varga, A., Flynn, D., Griffin, K., et al. (2016). Biodiversity 447 as a multidimensional construct. Proc. R. Soc. B Biol. Sci., 283, 20153005. 
Oliver, T.H., Heard, M.S., Isaac, N.J.B., Roy, D.B., Procter, D., Eigenbrod, F., et al. (2015).

Owens, I.P.F., Bennett, P.M. \& Harvey, P.H. (1999). Species richness among birds: Body size, life history, sexual selection or ecology? Proc. R. Soc. B Biol. Sci., 266, 933-939.

452 Pigot, A.L., Sheard, C., Miller, E.T., Bregman, T.P., Freeman, B.G., Roll, U., et al. (2020). Macroevolutionary convergence connects morphological form to ecological function in birds. Nat. Ecol. Evol., 4, 230-239.

R Core Team. (2018). R: A language and environment for statistical computing. Available for

Pigot, A.L., Tobias, J.A. \& Jetz, W. (2016). Energetic constraints on species coexistence in birds.

Rabinowitz, D. (1981). Seven forms of rarity. In: The biological aspects of rare plant conservation. Wiley, Chichester, UK, pp. 205-217.

461 Reich, P.B., Tilman, D., Isbell, F., Mueller, K., Hobbie, S.E., Flynn, D.F.B., et al. (2012). Impacts of biodiversity loss escalate through time as redundancy fades. Science, 336, 589-

464 Reinhardt, K., Kohler, G., Maas, S. \& Detzel, P. (2005). Low dispersal ability and habitat specificity promote extinctions in rare but not in widespread species: the Orthoptera of

467 Rosseel, Y. (2012). lavaan : an R package for structural equation modeling. J. Stat. Softw., 48, 136.

469 Rubolini, D., Liker, A., Garamszegi, L.Z., Møller, A.P. \& Saino, N. (2015). Using the birdtree.org website to obtain robust phylogenies for avian comparative studies: a primer. 
Curr. Zool., 61, 959-965.

472 Şekercioğlu, Ç.H., Daily, G.C. \& Ehrlich, P.R. (2004). Ecosystem consequences of bird declines. Proc. Natl. Acad. Sci. U. S. A., 101, 18042-18047.

474 Sheard, C., Neate-Clegg, M.H.C., Alioravainen, N., Jones, S.E.I., Vincent, C., MacGregor, H.E.A., et al. (2020). Ecological drivers of global gradients in avian dispersal inferred from

Soliveres, S., Van Der Plas, F., Manning, P., Prati, D., Gossner, M.M., Renner, S.C., et al. (2016). Biodiversity at multiple trophic levels is needed for ecosystem multifunctionality.

Sukumaran, J. \& Holder, M.T. (2010). DendroPy: A Python library for phylogenetic computing. Bioinformatics, 26, 1569-1571.

Tilman, D., Isbell, F. \& Cowles, J.M. (2014). Biodiversity and ecosystem functioning. Annu. Rev. Ecol. Evol. Syst., 45, 471-493.

Tilman, D., Knops, J., Wedin, D., Reich, P., Ritchie, M. \& Siemann, E. (1997). The influence of

Tobias, J.A. AVONET: A global database of bird traits. Ecol. Lett. Submitted for publication in the same special section within this issue.

488 Tobias, J.A., Ottenburghs, J. \& Pigot, A.L. (2020). Avian diversity: speciation, macroevolution 489 and ecological function. Annu. Rev. Ecol. Evol. Syst., 51.

490 Tobias, J.A. \& Pigot, A.L. (2019). Integrating behaviour and ecology into global biodiversity 491 conservation strategies. Philos. Trans. R. Soc. B Biol. Sci., 374, 20190012.

492 Villéger, S., Mason, N.W.H. \& Mouillot, D. (2008). New multidimensional functional diversity 493 indices for a multifaceted framework in functional ecology. Ecology, 89, 2290-2301. 
494 Webb, C.O., Ackerly, D.D., McPeek, M.A. \& Donoghue, M.J. (2002). Phylogenies and

495 community ecology. Ann. Rev. Ecol. Evol. Syst., 33, 475-505.

496 Weeks, B.C. \& Claramunt, S. (2014). Dispersal has inhibited avian diversification in

497 Australasian archipelagoes. Proc. R. Soc. B Biol. Sci., 281, 20141257.

498 Weeks, B.C., Claramunt, S. \& Cracraft, J. (2016a). Integrating systematics and biogeography to

499 disentangle the roles of history and ecology in biotic assembly. J. Biogeogr., 43, 1546-

$500 \quad 1559$.

501 Weeks, B.C., Gregory, N. \& Naeem, S. (2016b). Bird assemblage vulnerability depends on the 502 diversity and biogeographic histories of islands. Proc. Natl. Acad. Sci. U. S. A., 113, 10109-

$503 \quad 10114$

504 Wilman, H., Belmaker, J., Simpson, J., de la Rosa, C., Rivadeneira, M.M. \& Jetz, W. (2014).

505 EltonTraits 1.0: Species-level foraging attributes of the world's birds and mammals.

$506 \quad$ Ecology, 95, 2027-2027.

507 Winemiller, K.O., Fitzgerald, D.B., Bower, L.M. \& Pianka, E.R. (2015). Functional traits,

508 convergent evolution, and periodic tables of niches. Ecol. Lett., 18, 737-751. 




512 Figure 1. Conceptual illustration of the relationship between diversity and extinction risk. (a)

513 Species in more diverse assemblages are hypothesized to have reduced exposure to extinction

514 pressure as a result of biodiversity effects on ecosystem functioning and stability. (b) The

515 phenotypic and biogeographic attributes of individual species in an assemblage determine the

516 impacts of the extinction pressures to which they are exposed (i.e. their vulnerability). (c)

517 Together, the diversity and attributes of constituent species within an assemblage determine the

518 contemporary extinction risk of assemblages. Thus, the relationship between diversity and

519 extinction risk may depend on a trade-off between two inter-dependent processes: 1) the

520 reduction of extinction risk associated with higher assemblage diversity $(a \rightarrow c)$, and 2) the

521 consequent accumulation of vulnerable species in more diverse assemblages $(a \rightarrow b \rightarrow c)$. 




525 Figure 2. Relationship between bird diversity and assemblage vulnerability mapped at global

526 scales. Patterns shown are based on estimates of (a) taxonomic, (b) functional, and (c)

527 phylogenetic diversity calculated from all species mapped as occurring in 1 degree grid cells

528 worldwide. Functional diversity is estimated from morphological traits for over 10,000 bird

529 species. We use biogeographical, ecological and morphological attributes of all species to

530 generate an index of assemblage vulnerability (a metric of mean vulnerability to extinction for

531 species in an assemblage). Maps show each variable standardized to a mean of 0 and standard

532 deviation of 1; the logarithm of standardized functional richness estimates that were transformed

533 to be positive are mapped. 


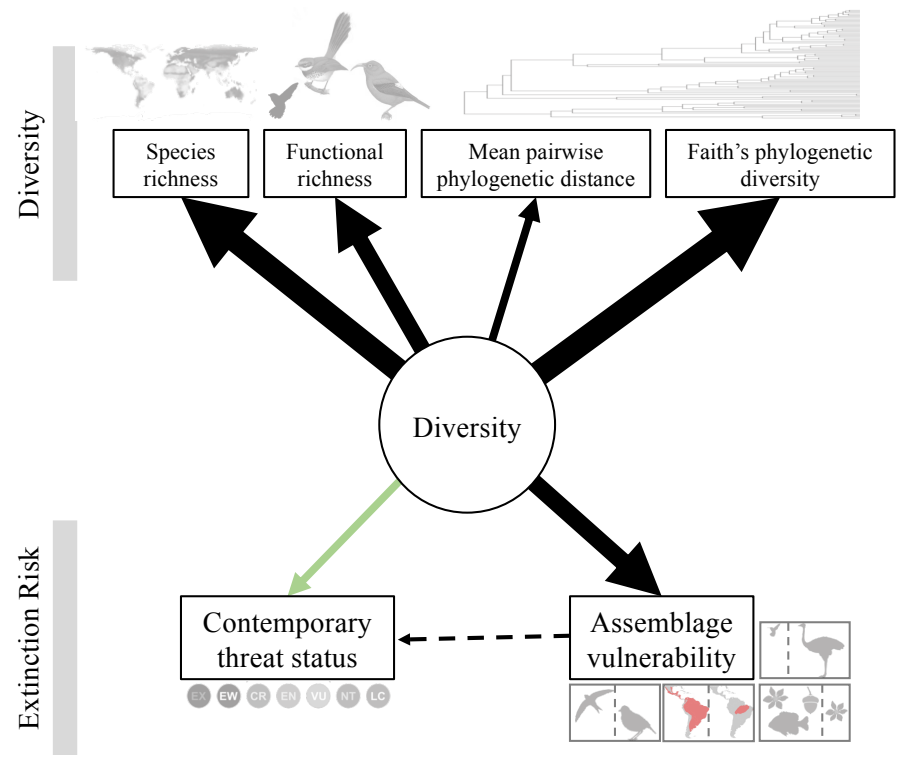

535 Figure 3. Diversity influences extinction risk in birds. We use structural equation modeling to 536 explore links between bird diversity, assemblage vulnerability, and contemporary threat status.

537 Black arrows indicate positive relationships; the green arrow indicates a negative relationship; all

538 relationships are significant. The width of the arrows is scaled to standardized effect size, solid

539 lines represent significant relationships and dashed lines represent relationships that are not

540 significant. We model bird assemblage diversity as a latent variable, measured using alternative

541 univariate dimensions (taxonomic, functional and phylogenetic diversity). The arrows

542 connecting diversity to univariate dimensions represent the loadings of diversity on each

543 dimension. Arrows connecting diversity, assemblage vulnerability, and contemporary threat

544 status represent regression parameters connecting the predictor to response variables. Diversity

545 has a strong positive relationship with assemblage vulnerability, suggesting that species

546 predisposed to extinction have accumulated in diverse assemblages. Via this pathway, diversity

547 has a positive effect on contemporary threat status although, importantly, this is an order of

548 magnitude weaker than the direct negative effect of diversity, and this relationship is not

549 significant (i.e. diversity reduces contemporary threat status overall). 